\title{
A enfermagem de saúde pública e as políticas públicas de saúde: um estudo de caso
}

\author{
Public health nursing and public health policies: a case study \\ La enfermería de salud pública y las políticas públicas de salud: uno estudio de caso
}

Carmen Maria dos Santos Lopes Monteiro da Cunha ${ }^{1,2}$ (1)

Maria Adriana Pereira Henriques ${ }^{2,3}$ (D) Andreia Jorge Silva Costa ${ }^{2,3}$ (1)

1. Universidade de Lisboa, Escola Superior de Enfermagem de Lisboa, Programa de Doutoramento em Enfermagem. Lisboa, Portugal.

2. Centro de Investigação, Inovação e Desenvolvimento em Enfermagem de Lisboa, Escola Superior de Enfermagem de Lisboa. Lisboa, Portugal.

3. Universidade de Lisboa, Faculdade de Medicina, Instituto de Saúde Ambiental. Lisboa, Portugal.
Autor correspondente:

Carmen Maria dos Santos Lopes Monteiro da Cunha.

E-mail: carmencunha@campus.esel.pt.

Recebido em 10/05/2021.

Aprovado em 02/06/2021.

\section{Resumo}

Objetivos: caracterizar as intervenções do enfermeiro especialista de saúde comunitária e de saúde pública de um Agrupamento de Centros de Saúde; identificar os principais fatores facilitadores para o desempenho das competências, e a contribuição para as políticas públicas de saúde. Método: estudo de caso, com enfermeiros da especialidade em enfermagem de saúde comunitária e de saúde pública. Coleta de dados por meio de entrevista, no período de outubro a dezembro de 2019. A análise foi guiada por duas estratégias: partir das proposições teoréticas e trabalhar os dados emergentes; e duas técnicas: combinar padrões, "pattern matching" e construir explicações, "explanation building". Resultados: a partir da matriz construída, surgiram dez proposições teoréticas, dez evidências emergentes, cinco padrões e cinco hipóteses explicativas. Sobressaem intervenções de vigilância epidemiológica, planeamento e gestão de projetos. A formação pessoal e a comunicação destacam-se como fatores facilitadores. Conclusão e implicações para a prática: a intervenção do enfermeiro dessa especialidade apresenta uma dimensão centrada no planeamento e gestão de projetos, identifica e reflete os fatores facilitadores do desempenho das suas competências, enquanto aplicador de políticas públicas de saúde.

Palavras-chave: Enfermagem de Saúde Pública; Enfermeiros de Saúde Pública; Saúde Pública; Políticas Públicas de Saúde; Estudo de Caso.

\section{Abstract}

Objectives: to characterize the interventions of specialist nurses of community health and public health of a Group of Health Centers, to identify the main facilitating factors for the performance of competences, and the contribution to public health policies. Method: this is a case study carried out with nurses specialized in community health and public health nursing. Data were collected through interviews from October to December 2019. Analysis was guided by two strategies: starting from the theoretical propositions and working the data from the ground up; and two techniques: pattern matching and explanation building Results: from the constructed matrix, ten theoretical propositions, ten emerging evidences, five patterns and five explanatory hypotheses emerged. There are interventions for epidemiological surveillance, planning and project management. Personal training and communication stand out as facilitating factors. Conclusion and implications for practice: the intervention of the nurses of these specialties presents a dimension centered on project planning and management identifies and reflects the facilitating factors of the performance of their competencies, as an applicator of public health policies.

Keywords: Public Health Nursing; Public Health Nurses; Public Health; Public Health Policy; Case Study.

\section{Resumen}

Objetivos: caracterizar las intervenciones del enfermero especialista en salud comunitaria y salud pública de un Grupo de Centros de Salud, identificar los principales fatores facilitadores para el desempeño de competencias, y la contribución a las políticas públicas de salud. Método: estudio de caso, con enfermeros especialistas en enfermería de salud comunitaria y de salud pública. Recolección de datos realizada por entrevista, de octubre a diciembre de 2019. El análisis estuvo guiado por dos estrategias: partiendo de las proposiciones teóricas y trabajando con los datos emergentes; y dos técnicas: combinación de patrones, "pattern matching" y construcción de explicaciones, "explanation building". Resultados: De la matriz construida surgieron diez proposiciones teóricas, diez evidencias emergentes, cinco patrones y cinco hipótesis explicativas. Se destacan intervenciones de vigilancia epidemiológica, planificación y gestión de proyectos. El entrenamiento personal y la comunicación se destacan como factores facilitadores. Conclusión e implicaciones para la práctica: La intervención del enfermero en estas especialidades presenta una dimensión centrada en la planificación y gestión de proyectos, identifica y refleja los factores facilitadores del desempeño de sus competencias, como aplicador de las políticas públicas de salud.

Palabras clave: Enfermería de Salud Pública; Enfermeras de Salud Pública; Salud Pública; Políticas Públicas de Salud; Estudio de Caso. 


\section{INTRODUÇÃO}

Este estudo enfoca a ação do enfermeiro especialista de saúde comunitária e de saúde pública, enquanto ator coparticipante na definição das políticas públicas de saúde, tendo como contexto, o caso de um Agrupamento de Centros de Saúde (ACES). Trata-se de uma área pouco estudada e valorizada, como concluímos através de uma revisão de literatura ${ }^{1}$. No ano de 2020, ano internacional do enfermeiro, importa realçar que ele está especialmente bem colocado para aconselhar as pessoas a fazerem escolhas mais saudáveis, porque estabelece uma relação terapêutica eficaz pela proximidade ${ }^{2}$.

Conforme a Associação Americana de Saúde Pública, Secção de Enfermagem de Saúde Pública3:2, "enfermagem de saúde pública é a prática de promoção e proteção da saúde das populações, recorrendo aos conhecimentos da enfermagem, social e ciências de saúde pública". A Ordem dos Enfermeiros de Portugal refere que:

a Enfermagem propõe novas visões sobre os cuidados de Saúde em Portugal, que se devem centrar na proximidade aos doentes e suas famílias, na supervisão e formação de profissionais cada vez mais capacitados, na reorganização dos cuidados de Saúde na comunidade e na consequente rentabilização de recursos humanos e materiais ${ }^{4: 1}$.

Das atuais políticas públicas de saúde, do XXII Governo português, destacamos que, valoriza a saúde pública como área de intervenção, a boa gestão dos sistemas de alerta e resposta atempada dos serviços, o diagnóstico de situações problemáticas e elaboração de planos estratégicos de ação com a comunidade, priorizando respeito pelos profissionais de saúde em novos modelos de cooperação, mesmo no que se refere à repartição de competências e responsabilidades 5 . Destaca-se a competência da colaboração na vigilância epidemiológica, no âmbito das suas competências nomeadamente através do Sistema Nacional de Vigilância Epidemiológica (SINAVE) 6 .

O presente trabalho tem como problema de pesquisa, a intervenção do enfermeiro especialista de saúde comunitária e de saúde pública de um ACES da região de Lisboa no contexto da contribuição para as políticas públicas de saúde. Traçamos como objetivos, caracterizar as intervenções do enfermeiro especialista de saúde comunitária e de saúde pública de um ACES, identificar os principais fatores facilitadores para o seu desempenho e a contribuição para as políticas públicas de saúde. Este estudo justifica-se, no sentido da busca pela relevância das intervenções de enfermagem no contexto específico da saúde pública, destacando as mais efetivas, não só para que o decisor político possa optar por elas, em tempos de constrangimentos de recursos públicos ${ }^{1}$, mas também para que o enfermeiro evidencie os seus conhecimentos e competências na sua área de intervenção.

\section{MÉTODO}

\section{Tipo do estudo}

Trata-se de uma abordagem qualitativa através de um estudo de caso $^{7}$, dedicado ao conhecimento do fenômeno complexo na sua profundidade ${ }^{8}$. Este é usado numa investigação, e constitui-se no método a escolher, quando o fenômeno em estudo não é facilmente distinguível do seu contexto. Recorre a múltiplas fontes de evidências, para a coleta de dados, como a análise documental, a observação direta, entrevistas, entre outras, com o propósito de aumentar a sua compreensão. Para este estudo recorremos apenas a entrevistas. Centra-se nas circunstâncias, dinâmicas e complexidades de um caso único, ou de um número pequeno de casos, em que se descrevem intervenções num contexto concreto ${ }^{7}$, como é o caso.

\section{População e Cenário}

A opção recai no caso único, holístico ou global, de um ACES da região de Lisboa que integra a administração indireta do Estado dotado de autonomia administrativa, financeira e patrimonial com vista a garantir à população residente na área geográfica atribuída, o acesso aos cuidados de saúde de qualidade através dos recursos disponíveis ajustados às necessidades de saúde da população, de acordo com o estabelecido no Plano Nacional de Saúde ${ }^{9}$ e, especialmente, concretizado no Plano Local de Saúde ${ }^{10}$. Caracteriza-se pelo ambiente geográfico coeso, população estável, diferenciada por um nível social elevado e escolarização relevante, indicadores de saúde notórios, com uma taxa de natalidade acima da média nacional, e de mortalidade abaixo da média nacional.

O ACES, para além das Unidades de Saúde Familiar, Unidade de Cuidados de Saúde Personalizados, Unidade de Cuidados na Comunidade, integra também uma Unidade de Saúde Pública. A esta compete, na área geodemográfica do ACES em que se inclui, o exercício conjunto de atribuições, previstas no arto 12 , no 1, do Decreto-Lei no 28/2008, de 22 de fevereiro ${ }^{11}$, elaborar informação e planos em domínios da saúde pública, proceder à vigilância epidemiológica, gerir programas de intervenção no âmbito da prevenção, promoção e proteção da saúde da população em geral, ou de grupos específicos, colaborar, de acordo com a legislação respetiva, no exercício das funções de autoridade de saúde.

Este estudo tem vindo a desenvolver-se desde o segundo semestre de 2019, num ACES com cerca de 160 enfermeiros. Foram estabelecidos, como critério de inclusão, todos os enfermeiros especialistas de saúde comunitária e de saúde pública do ACES, e de exclusão, os enfermeiros que se encontrassem de licenças médicas de longa duração. Resultou num total de 31 enfermeiros, sendo que todos aceitaram participar, não houve exclusões. A coleta de dados, na forma de entrevista, ocorreu entre 21 de outubro a 19 de dezembro de 2019.

\section{Coleta de dados}

A entrevista foi a opção selecionada entre várias fontes de evidências mais utilizadas no estudo de caso ${ }^{7}$. Os contatos 
prévios foram feitos por e-mail, para agendar dia, hora e local no local de trabalho. Com cada participante foi gravada uma entrevista, com cerca de 40 minutos. Após as transcrições das entrevistas, procedeu-se à solicitação da respectiva validação por parte dos entrevistados, também por e-mail. A entrevista baseou-se na aplicação de um protocolo de entrevista semiestruturada, composto por 31 questões abertas. Acresce ainda a consulta documental das políticas públicas de saúde, nos sites oficiais de divulgação das mesmas, nomeadamente governamentais.

Recorremos a todas as entrevistas $(n=31)$, efetuadas pela primeira autora, enquanto doutoranda, que cursou disciplina curricular específica para tal, e não conhecia pessoalmente os entrevistados, nem eles a conheciam, e foi feito um agendamento com breve apresentação.

\section{Análise e tratamento dos dados}

A organização dos dados para análise realizou-se com base numa matriz, composta por duas estratégias e duas técnicas de análise, propostas pelo autor, para o estudo de $\mathrm{caso}^{7}$. A estratégia inicial decorre das proposições teoréticas prévias, que dirigem o caso em concreto, e a segunda estratégia visa trabalhar os dados emergentes. A estas, são adicionadas duas técnicas de análise, combinar padrões, "pattern matching", e construir explicações, "explanation building"7.

A aplicação dessa matriz operacionalizou-se através da recolha dos dados pertinentes nas entrevistas, após elaboração sistematizada de planilhas em Excel, com todos os dados obtidos, recorrendo também ao software de análise qualitativa MAXQDA2020, que nos apoiou na codificação dos dados e na criação de categorias de análise.

A primeira estratégia baseia-se no fato de que as proposições delinearam o plano de coleta dos dados, conduzindo, assim, a prioridades analíticas, e a segunda contrasta diretamente com esta, já que, em vez de pensar em quaisquer proposições teoréticas, simplesmente convida a "passear entre os dados", ou a "jogar com eles".

A primeira técnica analítica compara padrões baseados em dados empíricos com os previstos, esperados, antes mesmo da coleta dos dados ${ }^{7}$. Para tal recorremos a hipóteses formuladas, com base numa Revisão Sistemática de Literatura (RSL) ${ }^{12-14}$, por nós efetuada, e com resultados publicados, com a questão de investigação: "O que existe publicado sobre competências e intervenções do enfermeiro de saúde comunitária e de saúde pública?"12-14. Segundo o autor ${ }^{7}$, trata-se de procurar os "comos (how's)" e os "porquês (why's)", no sentido de os verificar ou infirmar. Ao passo que a segunda técnica analítica, construir explicações, tem como fim analisar o estudo de caso através da construção de uma explicação do mesmo, no encalço do autor ${ }^{7}$.

As políticas públicas de saúde são abordadas descritivamente, a partir das fontes. A respetiva análise documental foi realizada com a leitura dos textos oficiais, e seleção dos pertinentes ao caso em estudo.

\section{Aspetos éticos}

Foi obtido o parecer favorável da Comissão de Ética para a Saúde, da Administração Regional de Saúde, de Lisboa e Vale do Tejo, por deliberação em 10 de maio de 2019, com a referência 4547/CES/2019. Para atender os princípios éticos de proteção dos participantes, foram elaborados, apresentados e aceites, individualmente, os Consentimentos, Informados, Livres e Esclarecidos, antes da realização de cada entrevista, bem como a garantia da confidencialidade e anonimato dos mesmos. Com vista à proteção das informações, sem o registro de dados pessoais, optou-se pela utilização de um código alfanumérico. Considerando o propósito acadêmico e científico, foram também obtidos os consentimentos dos participantes, para a apresentação das suas perspectivas nos resultados do estudo e nas respectivas publicações.

\section{RESULTADOS}

Os participantes são enfermeiros, na maioria do sexo feminino e com uma média de idade de 48 anos. Das entrevistas, algumas se mostram, particularmente, relevantes para os objetivos deste estudo.

Com base na matriz descrita, organizamos em quatro grupos, correspondendo cada um a uma das estratégias, ou técnicas de análise.

I. O primeiro grupo resulta da primeira estratégia de análise. Trata-se do recurso às proposições teoréticas, desdobradas dos dois objetivos, e concretizadas em questões que constavam do guia das entrevistas. Organizamos os primeiros resultados, emergentes das dez proposições teoréticas, que, a seguir, elencamos.

1‥ Proposição - Atividades que enformam as intervenções do enfermeiro.

[...] responsável pelo SINAVE, participação, gestão, monitorização e avaliação dos projetos (E05); [...] participação, elaboração, acompanhamento, implementação dos planos (formação, gestão das vacinas) (E02, E08); [...] participação, gestão de grupos, participação em programas; reuniões de parcerias (E01).

2a. Proposição - Contribuição do enfermeiro para a programação da unidade.

Para a equipe (multidisciplinar) (E01, E02, E05); [...] para trabalhos e projetos; com a visão da enfermagem (E01); [...] propostas de projetos, os indicadores (projetos) são da equipe (E05); [...] com o núcleo dos projetos (E08).

3a. Proposição - A prática do enfermeiro.

Gestão de informações (E01); [...] gestão de atividades dos projetos, verificação dos dados dos pacientes, articular 
com equipamentos de saúde (E02); [...] articular com outros colegas, articular com os pacientes, investigações através da consulta do processo clínico (E05); [...] apoiar, colaborar e orientar os colegas, organizar procedimentos preventivos (E08).

4a . Proposição - A importância da intervenção do enfermeiro.

Competências, know how (E01); [...] área dos idosos, área da saúde escolar, todo o ciclo de vida (E02); [...] vigilância epidemiológica da comunidade, contribui para completar o todo (E05).

5a․ Proposição - Os problemas e dilemas do enfermeiro.

A comunicação, a estrutura da unidade, a falta de procedimentos bem definidos, a falta de acessos às plataformas (E01); [...] a parte de informática, comunicação com o computador, problemas com telecomunicações (E02); [...] falta de um enfermeiro responsável pela enfermagem (E05); [...] mudança cria dilemas e constrangimentos (E08);

6ạ. Proposição - Benefícios e vantagens da presença do enfermeiro.

A visão holística, olhar de uma forma mais alargada, mais global (E01); [...] know how e experiência profissional (E02); [...] formação de base e específica (E05); [...] todo um processo que tem que passar pela enfermagem (E08).

7a. Proposição - Fatores facilitadores para o enfermeiro.

Permanentemente, investigar novos conhecimentos, novas competências, fazer omeu trabalho de casa (E01); [...] melhor equipamento, flexibilidade de horário, equipe multidisciplinar com mais pessoas (E02); [...] boa comunicação entre nós, condições físicas do trabalho, a liderança (E05); [...] noção clara das nossas competências (E08).

8a. Proposição - Fatores que dificultam a ação do enfermeiro.

Não ser valorizado pelos pares, e outros profissionais (E01); [...] a inexperiência, impacto de situação nova, dificuldades do funcionamento da própria organização, dificuldades organizacionais (E02); [...] falta de um coordenador de enfermagem próximo (E05); [...] desadequação entre $o$ esperado e o real (o esperado e as reais competências) (E08).

9a. Proposição - Mudanças para ajudar o enfermeiro.

Centrar-se mais no aumentar os seus conhecimentos (E01); [...] melhorar o sistema e o apoio informático (E02);
[...] criar uma estratégia, manuais de procedimentos e de informação, haver manuais de procedimentos escritos (E05); [...] mais formação nas áreas em que estão a trabalhar, mais recursos humanos e financeiros (E08).

10‥ Proposição - Alterações organizacionais.

Valorizar mais a saúde pública (E01); [...] mais encontros, jornadas para trocas de experiências; mais recursos humanos (E02); melhor definição dos projetos e intervenção de cada profissional, reuniões para avaliação e apresentação de propostas, haver enfermeiro chefe, ir para o terreno avaliare implementar medidas, divulgar mais nas outras unidades a saúde pública (E05); [...] manter a população informada; planejar ensinos na comunidade; maior intervenção na comunidade (E08).

II. O segundo grupo resulta da segunda estratégia, a análise dos dados emergentes, de onde sobressaem novos dados, diretamente relacionados com as mesmas questões teoréticas.

1‥ As atividades do enfermeiro articulam-se com a coordenação médica, podem ser alteradas. O enfermeiro realiza atividades no seio da equipe em que está integrado (E05).

2a. A contribuição do enfermeiro dilui-se na equipe multidisciplinar, o que dificulta avaliar as atividades concretas do enfermeiro (E05), e tem um papel fulcral na saúde pública, sem grande poder de decisão (E08).

3a. Há alterações aos planos do enfermeiro (E02), que o podem deixar descontente, sobretudo se o limitam ao trabalho à secretária, desejando ir à comunidade fazer investigação no terreno (E05). O enfermeiro tem um protocolo, porque é importante todos seguirem a mesma coisa (E08).

4‥ É importante o que o enfermeiro é, e a sua história (E01). Seria vantajoso o enfermeiro ir à comunidade realizar a continuidade do seu estudo. As atividades do enfermeiro poderiam ser mais valorizadas e aproveitadas (E05), e vai ao encontro dos objetivos, do que se espera (E08).

5 a. Oenfermeiro sente que recursos materiais desadequados levam ao mal aproveitamento dos recursos humanos (E02).

6⿳. O enfermeiro com as suas competências e qualidades enriquece muito a unidade, com uma sensibilidade para avaliar e cuidar de uma forma diferente (E05).

7ª . Facilitaria a existência de um enfermeiro coordenador para a definição de funções nos projetos (E05), e intervir de forma articulada em cada situação (E08). 
8‥ A falta de valorização poderá dificultar o papel do enfermeiro (E01). Dificuldades de recursos de informação fazem perder tempo (E02). A inexistência de um enfermeiro responsável, coordenador, pode dificultar a articulação com o coordenador médico, e com a equipe (E05), bem como o reconhecimento da área geodemográfica (E08).

9a. Facilitaria haver equipes de execução com identificação das pessoas concretas (E05), poder conhecer todos os projetos e ações (E08).

10‥ Alteraria, a divulgação e o impacto (E01), as equipes cada vez mais reduzidas (E02), e o desenvolvimento da ação de saúde pública (E05). O enfermeiro sente que poderia ir mais para o terreno avaliar e implementar medidas (E08).
III. O terceiro grupo forma-se a partir da primeira técnica de análise, combinar padrões, "pattern matching", recorrendo a cinco padrões desenhados com base na RSL ${ }^{12-14}$. Foram estes os escolhidos, por serem os mais representativos, sendo confrontados com os que resultam das evidências dos dados empíricos, no Quadro 1.

IV. O quarto grupo tem por base a segunda técnica de análise, construir explicações, "explanation building". Destina-se a explicar o fenómeno através de uma sequência causal presumida, do mesmo, o como (how) e o porquê (why) alguns resultados ocorrem.

Face aos resultados e padrões obtidos, construímos cinco hipóteses explicativas e respectiva correspondência aos dados, apresentados no Quadro 2.

Quadro 1. Relação entre os padrões resultantes da RSL e os dados empíricos.

\section{Padrões}

1. O principal empenho da maior parte dos enfermeiros de saúde pública centra-se na vacinação.

2. A maior parte dos serviços é prestado a nível individual e não comunitário.

3. Os enfermeiros de saúde pública estão envolvidos em muitas atividades, para prevenir doenças e contribuir para a saúde das populações. No entanto, as suas atividades nem sempre são visíveis para o público e para o decisor político.

4. A necessidade de um modelo diferente de serviços de saúde à comunidade, que implemente a colaboração e encontre denominadores comuns entre os membros da equipa de saúde pública.

5. A demonstração de uma aliança frutuosa, entre o trabalho académico e os profissionais no sentido de conseguirem prestar serviços eficazes, de qualidade, bem como a existência de novos recursos.

\section{Dados empíricos}

1. Não encontramos qualquer correspondência ao primeiro padrão, relativo à vacinação, apuramos que se trata de uma atividade entre outras, como adiante fundamentaremos.

2. O mesmo aconteceu no que se refere ao segundo padrão, pois não obtivemos dados a confirmar a prestação de serviços individualizados, mais do que comunitários.

3. Para o terceiro padrão já encontramos combinações com os nossos dados empíricos, assumindo as seguintes dimensões:

[...] a contribuição do enfermeiro se dilui na equipe, o enfermeiro está envolvido em muitas atividades, na falta de reconhecimento pelos pares e de valorização do papel do enfermeiro, falta de reconhecimento e falta de divulgação pública das intervenções do enfermeiro (E01).

4. Relativamente ao quarto padrão, sobressaem diversas evidências nos nossos dados, em diferentes formulações: o enfermeiro não se sente bem se ficar limitado ao trabalho à secretária (E05); daí a vontade de que o modelo inclua mais a presença e intervenção do enfermeiro na comunidade (E05, E08); expressa a necessidade de um enfermeiro coordenador, por o modelo existente apenas prever um coordenador médico (E05); inclusão de mais recursos humanos e definição de funções (E02); manuais de procedimentos (E05); proposta de várias alterações, como encontros e jornadas (E02).

5. O quinto padrão diz respeito aos fatores facilitadores para o desempenho das competências: verificamos que também o enfermeiro sublinha a importância da atualização contínua (E01), mais formação (E08), mas, sobretudo, mais e melhores recursos humanos e materiais (E02, E08). 
Quadro 2. Hipóteses explicativas e dados empíricos.

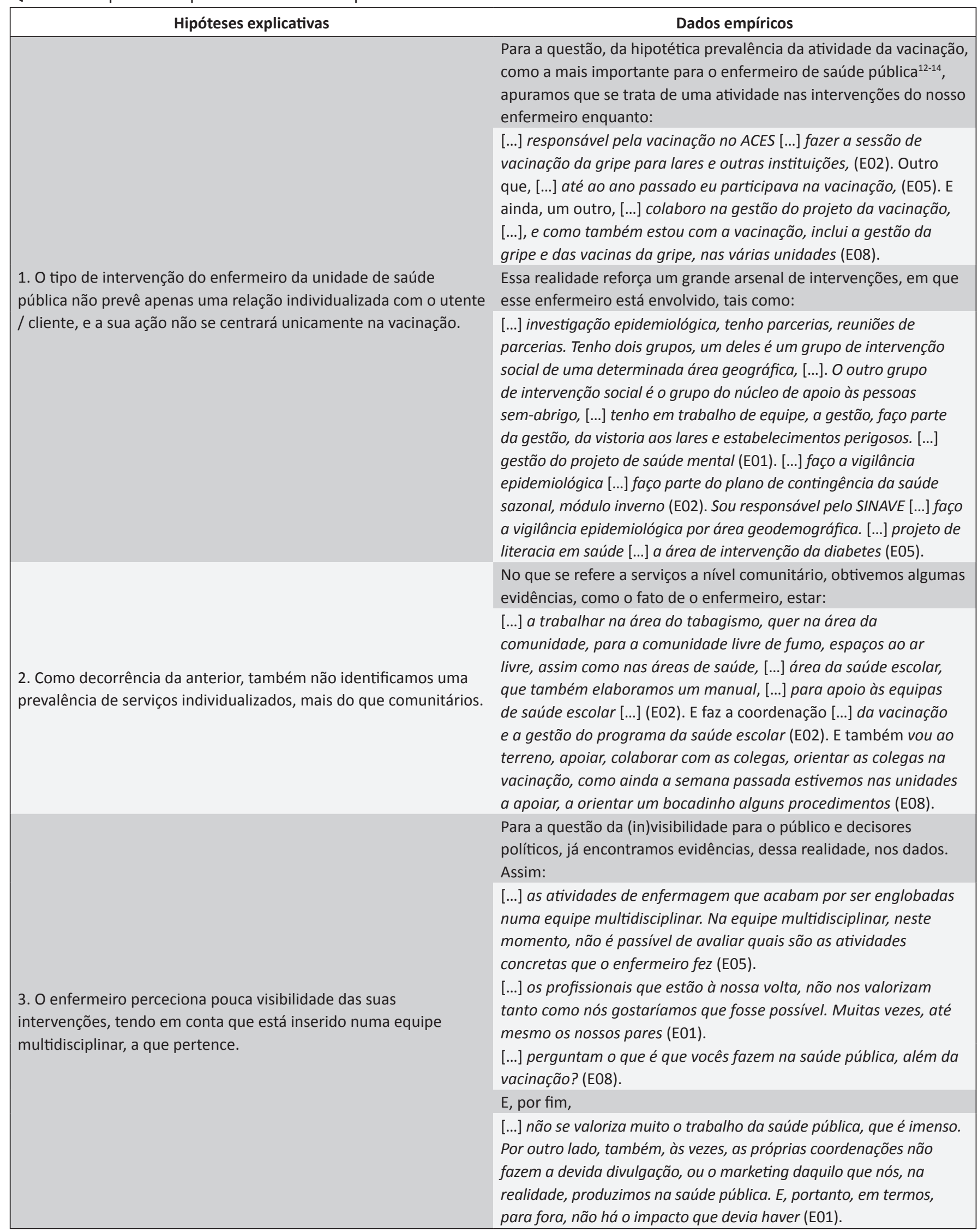


Quadro 2. Continuação...

\begin{tabular}{|c|c|}
\hline Hipóteses explicativas & Dados empíricos \\
\hline \multirow{5}{*}{$\begin{array}{l}\text { 4. O enfermeiro está disponível para ver alterado o seu modelo } \\
\text { de atuação, no sentido de estar mais no terreno, e voltado para a } \\
\text { comunidade, mas também na dimensão organizacional, com um } \\
\text { enfermeiro coordenador, definição de funções, recursos humanos e } \\
\text { manuais de procedimentos. }\end{array}$} & $\begin{array}{l}\text { Sobre a implementação de um modelo diferente de serviços à } \\
\text { comunidade, vários depoimentos ilustram essa vontade: }\end{array}$ \\
\hline & E, no que diz respeito aos recursos humanos, \\
\hline & $\begin{array}{l}\text { [...] as equipes são cada vez mais reduzidas para as áreas } \\
\text { geográficas que nós temos (...) nós não conseguimos nem dar } \\
\text { resposta ao trabalho que temos diariamente (E02). [...] muitas } \\
\text { vezes, quando se tem que intervir, a nível, para fora da saúde } \\
\text { pública, às vezes, era importante que falássemos todos a mesma } \\
\text { linguagem e, às vezes, isso não acontece (E01). }\end{array}$ \\
\hline & $\begin{array}{l}\text { É ainda notada, e aparentemente desejada, a presença de um } \\
\text { coordenador de enfermagem, assim, }\end{array}$ \\
\hline & $\begin{array}{l}\text { [...] punha um quinto elemento de enfermagem, para haver uma } \\
\text { chefe interna. Esta é a estratégia número um, para mim (E05). }\end{array}$ \\
\hline \multirow[b]{2}{*}{$\begin{array}{l}\text { 5. O enfermeiro tem consciência de que é importante continuar } \\
\text { a sua formação, mas também reconhece que outros fatores } \\
\text { facilitam o seu desempenho, como boa comunicação, funções bem } \\
\text { definidas, melhores instalações e condições de trabalho. }\end{array}$} & Relativamente aos fatores facilitadores de desempenho: \\
\hline & $\begin{array}{l}\text { [...] uma das coisas muito importantes no desempenho é o não } \\
\text { pensar que aqueles conhecimentos que tem são suficientes (E01). } \\
\text { [...] contribui melhor equipamento, flexibilidade de horário, equipes } \\
\text { multidisciplinares, que tivéssemos mais pessoas, e ainda uma } \\
\text { boa comunicação entre nós (E02). [...] é muito importante definir } \\
\text { funções, elaborar manuais de procedimentos (...), e depois dar } \\
\text { visibilidade do trabalho (E05). } \\
\text { [...] se eu tiver interiorizado bem as minhas competências, em cada } \\
\text { área eu vou dar a resposta certa (E08). }\end{array}$ \\
\hline
\end{tabular}

\section{DISCUSSÃO}

Sobressai dos resultados que apresentamos, partindo das proposições teoréticas e dos dados emergentes, um "retrato" da enfermagem de saúde pública, levada à prática pelo enfermeiro especialista de saúde comunitária e de saúde pública. Este desenvolve uma pluralidade de intervenções, que representa como relevantes, ainda que nem sempre, aparentemente, tenha autonomia na sua definição. Enfrenta algumas dificuldades, como procedimentos de intervenções melhor definidos, ou mesmo organizacionais, como a ausência de um enfermeiro chefe. Reconhece benefícios e vantagens da sua presença, resultantes da sua visão holística e da sua formação específica, que facilitam o seu desempenho. Não se sentindo suficientemente valorizado, na sua atuação e reconhecimento, quer social, quer pelos seus pares, aponta estratégias, como definir funções e papéis de cada um, na equipe multidisciplinar que integra. Mas também, a nível de divulgação do seu trabalho, e investimento em recursos humanos e materiais, que permitam ao enfermeiro ir mais para o terreno implementar e avaliar medidas.

Esse "retrato" parece algo diferente do que está previsto para essa área de enfermagem, em outros sistemas de saúde, como é o caso do Reino Unido², com domínios de atuação, como a promoção de saúde e prevenção de mortalidade precoce. Mas, percebe-se situar-se em linha com outras realidades, onde o enfermeiro também busca a afirmação do seu estatuto, desafiando o "status quo" atual, por mudanças estruturais que possibilitem a sua liderança repartida, no sistema de saúde ${ }^{15}$.

Partimos, depois, dos padrões desenhados, com base na RSL, na modalidade scoping review ${ }^{1}$, já publicada. Face aos padrões que emergiram desta revisão, construímos as nossas hipóteses explicativas, no sentido de aprofundar a análise dos dados, construindo uma possível explicação do caso em análise, neste estudo de caso. Com base nos seus como (how), e porquê (why), tentamos encontrar explicações para os resultados encontrados, através de um conjunto presumido de elos causais, sobre eles ${ }^{7}$. 
Em contraponto com a nossa primeira hipótese explicativa, de que o enfermeiro não parece ter apenas uma relação individualizada com o público / utente / cliente, nem apenas centrado na vacinação, um estudo nacional realizado em Israel ${ }^{16}$. Nele, terão participado cerca de $80 \%$ dos enfermeiros de saúde pública, e fornece uma descrição detalhada e atualizada do trabalho, e áreas de prática dos mesmos. Refere que um dos resultados chave é que, o principal empenho da maior parte dos enfermeiros de saúde pública se centra na vacinação.

Em conformidade com a nossa segunda hipótese explicativa, de não identificarmos uma prevalência de serviços individualizados, mais do que comunitários, um artigo realizado nos Estados Unidos ${ }^{17}$, com enfermeiros visitadores de saúde pública, tendo como principal objetivo descrever a ação desse enfermeiro na visitação domiciliária, no caso em concreto, para realizar intervenções nutricionais, apresenta uma enfermagem de saúde pública centrada, quer na comunidade, quer no indivíduo.

No mesmo sentido da nossa terceira hipótese, de que o enfermeiro perceciona pouca visibilidade das suas intervenções, até porque se diluem dentro da equipe multidisciplinar a que pertence, podemos realçar a posição de autores, num estudo da América do Norte ${ }^{18}$. Tendo resultado de dois inquéritos nacionais, com questões focadas na atividade do enfermeiro de saúde pública, para promover a respetiva visibilidade, identificando atividades e explorando o seu impacto na comunidade, no fundo, espelha o mesmo sentir. Afirma ser evidente que os enfermeiros de saúde pública fazem muitas atividades, para prevenir doenças e contribuir para a saúde das populações, e que não são sempre visíveis para o público e decisores políticos. Também porque muitas das suas intervenções, que fortalecem os determinantes sociais da saúde, são invisíveis.

Um artigo de Portugal, na modalidade de revisão da literatura publicada e não publicada, e análise documental, para analisar a composição da força de trabalho em saúde em Portugal, ilustra a questão da quarta hipótese explicativa, de uma evidência forte de que o enfermeiro pretende ver alterado o seu modelo de atuação, para estar mais no terreno e na comunidade, falta de um enfermeiro coordenador, e até de recursos humanos. Afirma-se nesse estudo existir algum espaço normativo, para ampliar as competências dos enfermeiros, no sentido de melhorar o desempenho do sistema de saúde. No entanto, chama a atenção que, tal mudança necessita de um consenso social, "e que, em Portugal, alguns parceiros manifestam reserva quanto à expansão do campo de atuação da profissão de enfermagem"19.

Em consonância com a nossa quinta hipótese, dos fatores que facilitam o seu desempenho, como boa comunicação, funções bem definidas, melhores instalações e condições de trabalho, citamos, a título de exemplo, um estudo israelita ${ }^{20}$. Aponta para uma aliança frutuosa entre investigação acadêmica e profissionais de saúde, a trabalhar juntos, no sentido de prestar serviços de alta qualidade aos utentes. Ou a publicação dos Estados Unidos ${ }^{21}$, que sustenta a existência de bases comuns, entre o nível das competências da enfermagem de saúde pública, e os princípios da aprendizagem em serviço: conhecimento experimental da comunidade; trabalhar com os parceiros chave e os decisores políticos; reflexão e conhecimento; profissionalismo e compaixão competências culturais; e engajamento cívico. Esses princípios, no desenvolvimento das experiências em saúde pública, aumentarão as probabilidades de sucesso.

Apresentamos, como possível explicação para o produto dos dados analisados, o tipo de intervenção prevista para este enfermeiro, que poderá ter um cariz mais próximo do planeamento, no importante controle dos dados epidemiológicos e clínicos dos utentes através de aplicações informáticas, como o SINAVE, usado para identificar situações de risco, recolher, atualizar, analisar e divulgar os dados referentes às doenças transmissíveis e outros riscos para a saúde pública. Bem como preparar planos de contingência para situações de emergência ou de calamidade pública ${ }^{6}$. E, ainda, o fato de se dedicar ao desenho e gestão de projetos, para além da interação com indivíduos, grupos e comunidades. Bem consciente que, a formação contínua e os meios à disposição, são os principais fatores facilitadores das suas competências.

Para as políticas públicas de saúde e o enfermeiro, importa salientar que, de acordo com Tedros A. Ghebreyesus, Diretor-Geral da Organização Mundial de Saúde, "Os enfermeiros são a espinha dorsal de todo o sistema de saúde: em 2020, convidamos todos os países a investirem em enfermeiros e parteiras como parte do seu compromisso com a saúde para todos"2:1.

Também no Brasil, uma publicação recente, chama a atenção para a importância do enfermeiro no desenvolvimento de ações de controle primário de doenças, como a tuberculose ${ }^{23}$. E a atual situação pandêmica, veio realçar a necessidade de planejar e colocar em ação recursos materiais e humanos em quantidade e qualidade suficientes, para suportar os serviços de saúde, em especial, no que diz respeito aos enfermeiros ${ }^{24}$, pondo mesmo em questão, a segurança desses profissionais ${ }^{25}$, em contraponto da sua indispensável contribuição.

Prevendo o programa do XXII Governo de Portugal ${ }^{5}$, na política de saúde, como política fundamental, recuperar o papel central da rede de cuidados de saúde primários, e até ampliar e melhorar a sua capacidade, na gestão dos recursos humanos, valorizando os profissionais de saúde, através de novas formas de cooperação e repartição de responsabilidades, nas profissões de saúde ${ }^{5}$, a atuação do enfermeiro, na prática, executa e reflete as políticas públicas de saúde. Mas, questiona se não deveria também ter uma participação efetiva, na feitura das mesmas.

Apesar de os números sugerirem que o enfermeiro terá pouca atuação política em Portugal, como se constatou no processo eleitoral que conduziu às últimas eleições legislativas, em 2019, onde quatro enfermeiros foram candidatos, ou seja, 2,27\% do total, e a previsão era de haver entre 2 a 5 enfermeiros, no total dos 230 deputados. Comprovamos a presença de um, na atual Assembleia da República, o órgão legislativo do país ${ }^{26}$.

A apoiar essa inquietação, a posição dos enfermeiros dos Açores, que já em 2016 sublinhavam, assumirem individual e coletivamente um trabalho informativo e de sensibilização, junto dos decisores políticos, para influenciar na definição de políticas 
de saúde ${ }^{27}$. Importa ainda realçar que, face às reformas em curso, no âmbito dos cuidados de saúde primários em Portugal, abremse novas oportunidades para o enfermeiro ganhar uma maior relevância, uma vez que, como defendem as autoras ${ }^{28}$, também em outros sistemas similares de saúde, está prevista, ou em prática, a possibilidade do desenvolvimento de competências alargadas para esse profissional.

\section{CONCLUSÃO E IMPLICAÇÕES PARA A PRÁTICA}

As intervenções identificadas do enfermeiro especialista de saúde comunitária e de saúde pública, parecem situar-se mais na vigilância epidemiológica, planeamento e gestão de projetos, com relevantes contributos para a saúde pública e para as políticas de saúde. Tem consciência dos fatores facilitadores para o desempenho das suas competências, e, tal como a maioria dos seus pares, é um executor de políticas públicas de saúde, vocacionado para ser também um ator no seu desenho e elaboração.

Como possível limitação, podemos apontar o fato de nos termos debruçado apenas sobre uma unidade de saúde. No entanto, os dados coletados e os resultados que emergem da análise, afiguram-se muito importantes para o conhecimento deste enfermeiro, dada a escassez de estudos sobre esta realidade, o que se traduz num avanço para a ciência da enfermagem, na sua dimensão de pesquisa e novo conhecimento, bem como alavancam uma investigação mais alargada, até no sentido de uma ampliação das suas competências.

Ainda que a coleta de dados do estudo se reporte a uma fase temporal, imediatamente anterior à atual pandemia (COVID-19), certamente não poderemos imaginar o seu combate, sem a relevante contribuição da enfermagem de saúde comunitária e de saúde pública, que, como parece emergir dessa investigação, dedica grande parte da sua atuação à vigilância epidemiológica.

\section{CONTRIBUIÇÕES DOS AUTORES}

Desenho do estudo: Carmen Maria dos Santos Lopes Monteiro da Cunha.

Aquisição de dados: Carmen Maria dos Santos Lopes Monteiro da Cunha, Maria Adriana Pereira Henriques, Andreia Jorge Silva Costa.

Análise de dados: Carmen Maria dos Santos Lopes Monteiro da Cunha, Maria Adriana Pereira Henriques, Andreia Jorge Silva Costa.

Interpretação dos resultados: Carmen Maria dos Santos Lopes Monteiro da Cunha, Maria Adriana Pereira Henriques, Andreia Jorge Silva Costa.

Redação e revisão crítica do manuscrito: Carmen Maria dos Santos Lopes Monteiro da Cunha, Maria Adriana Pereira Henriques, Andreia Jorge Silva Costa.

Aprovação da versão final do artigo: Carmen Maria dos Santos Lopes Monteiro da Cunha, Maria Adriana Pereira Henriques, Andreia Jorge Silva Costa.
Responsabilidade por todos os aspectos do conteúdo e a integridade do artigo publicado: Carmen Maria dos Santos Lopes Monteiro da Cunha, Maria Adriana Pereira Henriques, Andreia Jorge Silva Costa.

\section{EDITOR ASSOCIADO}

Aline Monroe (D)

\section{EDITOR CIENTÍFICO}

Marcelle Miranda da Silva (D)

\section{REFERÊNCIAS}

1. Cunha $C$, Costa A, Henriques MA. The public health nurse's interventions and responsibilities in Portugal: a scoping review. Rev Enferm UERJ. 2019;27:e37214. http://dx.doi.org/10.12957/reuerj.2019.37214

2. Wild K, McGrath M. Public health and health promotion for nurses at a glance. UK: Wiley Blackwell; 2019.

3. American Public Health Association, Public Health Nursing Section The definition and practice of public health nursing [Internet]. 2013 nov [citado 2020 jan 15]. Disponível em: https://www.apha.org

4. Ordem dos Enfermeiros. Participação dos enfermeiros na definição de políticas de saúde domina debate entre ministério, entidades e deputados da área da saúde [Internet]. 2013 [citado 2020 fev 3]. Disponível em: https://www.ordemenfermeiros.pt/arquivo-dep\%C3\%A1ginas-antigas/participa\%C3\%A7\%C3\%A3o-dos-enfermeirosna-defini\%C3\%A7\%C3\%A3o-de-pol\%C3\%ADticas-de-sa\%C3\%BAdedomina-debate-entre-minist\%C3\%A9rio-entidades-e-deputados-da$\%$ C3\%A1rea-da-sa\%C3\%BAde/

5. Portugal. Programa do XXII Governo Constitucional para a saúde [Internet]. 2019 [citado 2020 mar 10]. Disponível em: https://www.sns. gov.pt/institucional/politica-de-saude/

6. Direção-Geral da Saúde. SINAVE MED [Internet]. 2020 [citado 2020 mar 10]. Disponível em: https://www.dgs.pt/servicos-on-line1/sinavesistema-nacional-de-vigilancia-epidemiologica.aspx

7. Yin RK. Case study research and applications: design and methods 6th ed. Los Angeles: SAGE; 2018.

8. Creswell JW. Research design: qualitative, quantitative, and mixed methods approaches. 3th ed. Los Angeles: SAGE Publications; 2009.

9. Direção-Geral da Saúde. Plano Nacional de Saúde, revisão e extensão a 2020 [Internet]. 2015 [citado 2020 abr 5]. Disponível em: https://www. dgs.pt/a-dgs.aspx

10. Administração Regional de Saúde de Lisboa e Vale do Tejo. Plano Local de Saúde do ACES de Lisboa Ocidental e Oeiras [Internet]. 2016 [citado 2020 abr 5]. Disponível em: https://www.arslvt.min-saude.pt/uploads/ writer_file/document/1034/Plano_Local_Sa_de_v4-_Vers_o_Final.pdf

11. Portugal. Decreto-lei nำ $28 / 2008$ de 22 de fevereiro. Cria os agrupamentos de centros de saúde do Serviço Nacional de Saúde. Diário da República [periódico na internet], 2008: I Série (№ 38 de 22-02-2008): 1182-9 [citado 2020 abr 5]. Disponível em: https://dre.pt/pesquisa/-/search/247675/ details/maximized

12. Cunha $C$, Costa $A$, Henriques MA. Competências e intervenções do enfermeiro de saúde comunitária e de saúde pública: uma revisão de literatura. Atas CIAIQ2019 [Internet]. 2019; [citado 2020 abr 5];2:36170. Disponível em: https://proceedings.ciaiq.org/index.php/CIAIQ2019/ article/view/2038

13. Cunha $C$, Henriques MA, Costa A. Investigação e inovação em saúde: intervenções do enfermeiro de saúde comunitária e de saúde pública. Rev ROL Enferm [Internet]. 2020; [citado 2020 abr 5];43(Suppl 1):32-42. Disponível em: https://e-rol.es/rol_2020/IC_RESEARCH_INNOVATION_ DEVELOPMENT NURSING_2019.pdf

14. Cunha C, Costa A, Henriques MA. Community health and public health nursing: a systematic literature review. Gestão \& Saúde. 2020;11(2):8096. http://dx.doi.org/10.26512/gs.v11i2.29414 
15. Varghese J, Blankenhorn A, Saligram P, Porter J, Sheikh K. Setting the agenda for nurse leadership in India: what is missing. Int J Equity Health. 2018;17(1):98. http://dx.doi.org/10.1186/s12939-018-0814-0.

16. Haron Y, Honovich M, Rahmani S, Madjar B, Shahar L, Feder-Bubis P. Public health nurses' activities at a time of specialization in nursing-a national study. Public Health Nurs. 2019;36(1):79-86. http://dx.doi. org/10.1111/phn.12578.

17. Horning ML, Olsen JM, Lell S, Thorson DR, Monsen KA. Description of public health nursing nutrition assessment and interventions for home-visited women. Public Health Nurs. 2018;35(4):317-26. http:// dx.doi.org/10.1111/phn.12410.

18. Schaffer MA, Keller LO, Reckinger D. Public health nursing activities: visible or invisible? Public Health Nurs. 2015;32(6):711-20. http://dx.doi. org/10.1111/phn.12191.

19. Temido M, Dussault G. Papéis profissionais de médicos e enfermeiros em Portugal: limites normativos à mudança. Rev Port Saude Publica. 2014;32(1):45-54. http://dx.doi.org/10.1016/j.rpsp.2013.11.002.

20. Kagan I, Shachaf S, Rapaport Z, Livne T, Madjar B. Public Health Nurses in Israel: a case study on a quality improvement project of nurse's work life. Public Health Nurs. 2017;34(1):78-86. http://dx.doi.org/10.1111/phn.12261.

21. Brown CL. Linking public health nursing competencies and servicelearning in a global setting. Public Health Nurs. 2017;34(5):485-92. http://dx.doi.org/10.1111/phn.12330.

22. United Nations. Year of the nurse and the midwife highlights 'backbone' of health systems. Un News. [periódico na internet], jan 2020 [citado 2020 abr 5]. Disponível em: https://news.un.org/en/story/2020/01/1054531
23. Bertolozzi MR, Takahashi RF, França FOS, Hino P. A ocorrência da tuberculose e sua relação com as desigualdades sociais: estudo de revisão integrativa na base PubMed. Esc Anna Nery. 2020;24(1):e20180367. http://dx.doi.org/10.1590/2177-9465-EAN-2018-0367.

24. Soares SSS, Souza NVDO, Carvalho EC, Varella TCMML, Andrade KBS, Pereira SRM et al. De cuidador a paciente: na pandemia da Covid-19, quem defende e cuida da enfermagem brasileira? Esc Anna Nery. 2020;24(spe):e20200161. http://dx.doi.org/10.1590/2177-9465ean-2020-0161.

25. Santana N, Costa GA, Costa SSP, Pereira LV, Silva JV, Sales IPPM Segurança dos profissionais de saúde no enfrentamento do novo coronavírus no Brasil. Esc Anna Nery. 2020;24(spe):e20200241. http:// dx.doi.org/10.1590/2177-9465-ean-2020-0241.

26. Macedo MA. As legislativas vistas pela enfermagem [Internet]. Shifter Editorial; set 2019 [citado 2020 abr 2]. Disponível em: https://shifter. sapo.pt/2019/09/legislativas-enfermagem/

27. Pereira SM, Hernández-Marrero P. A decisão e desenvolvimento de políticas de saúde, o contributo central dos enfermeiros no desenvolvimento de políticas de saúde. Açoriano Oriental [periódico na internet], 26 jun 2016 [citado abr 4]. Disponível em: https://www.ordemenfermeiros.pt/ media/3723/junho16.pdf

28. Cunha C, Henriques MA, Costa A. A enfermagem de saúde pública: regulação e políticas públicas de saúde. Rev Bras Enferm. 2020;73(6):e20190550. http://dx.doi.org/10.1590/0034-7167-2019-0550. 